\title{
Personalized Support for Knowledge Sharing
}

\author{
Virginia Dignum \\ Center for Content and Knowledge Engineering \\ Institute of Information and Computing Sciences \\ Universiteit Utrecht \\ P.O. Box 80089, 3508 TB Utrecht \\ +31-30-2539492 \\ virginia@cs.uu.nl
}

\begin{abstract}
The realization that Knowledge Management (KM) is primarily a management science and not a computer science implies a different role for human-computer interaction in KM systems. This new role, supporting and extending human interaction, implies a need for intelligence-enhanced, integrated and personalized solutions. In this sense, $\mathrm{KM}$ requires the flexible integration of organizational and individual requirements and objectives, which can best be modeled using agent concepts. In this paper, we present an agent-based model for sharing that supports individual initiative and collaboration while prescribing a formal model for organizational processes. This model enables the development of user-oriented KM environments that focus on the collaboration between people.
\end{abstract}

\section{Keywords}

Knowledge Management, Collaboration, Software Agents, Personal Assistants

\section{INTRODUCTION}

Knowledge has been widely acknowledged as one of the determining factors for corporate competitiveness and advantage. In the past decade, we have witnessed an explosion of approaches to Knowledge Management (KM). $\mathrm{KM}$ is defined as a systematic, holistic approach to the sustainable improvement of the handling of knowledge on all levels of an organization [6]. Practitioners and business managers alike agree that issues of technology, process, people, and content must be addressed in KM to achieve success [8]. KM activities include the identification, acquisition, preservation, dissemination and use of enterprise knowledge to be able to respond to rapid changes in a knowledge-based economy.

Comprehensive $\mathrm{KM}$ endeavors recognize that $\mathrm{KM}$ is primarily a management science and not a computing science. A KM system that links to the real needs and goals of people on their real-world practices, and facilitates their contacts and interactions, has a much higher chance of success than one than will follow the 'official' workflow processes. This identifies a novel direction in KM, that of collaboration management. This shift in the focus of KM requires systems that meet the following requirements [5]:

- Assist people generate and apply 'just in time' and 'just enough' knowledge, prevent information overload and stimulate sharing of relevant knowledge in a dynamic, collaborative environment.

- Preserve individual autonomy and contribute to the creation of an atmosphere of trust between participants.

- Provide links individual action and company structure such that on one hand, innovative ways of doing things can be effectively integrated into company processes and, on the other hand, it can be verified whether actions are conform to company values and norms.

Agents offer a way to deal with complex systems that have multiple and distinct components, and are often used as a metaphor for autonomous, intelligent entities. Agent systems are therefore utmost qualified to model collaboration management systems. The agent modeling metaphor poses a major challenge to human-computer interaction since it drastically changes the way humans perceive and interact with a computer. Instead of directmanipulation, interaction is now perceived as delegation. In this paper, we present the consequences of an agent perspective inter-personal knowledge sharing. We demonstrate the applicability of these ideas in a KM case study.

\section{AGENT-MEDIATED KNOWLEDGE MANAGEMENT}

Taking a collaboration perspective on KM implies a different role for technology in KM, that of supporting and extending human interaction and learning, and therefore a need for intelligence-enhanced, integrated and personalized solutions. This is currently leading to an increasing interest in the use of multi-agent concepts for KM, mainly motivated by the fact that, like multi-agent systems, KM domains involve an inherent distribution of sources, problem solving capabilities and responsibilities [2]. In such domains, it is important on the one hand, to assure that activity conforms to (existing) organizational norms and aims at the realization of global goals, but, on the other hand, the autonomy of participants must be preserved, such that the organization can profit from individual characteristics and skills. Moreover, interactions in KM 
environments are sophisticated, including negotiation, information sharing and coordination, and require the complex social skills with which agents can be endowed. Furthermore, solutions for KM problems cannot be entirely prescribed from start to finish and therefore reactive and proactive models are required that can respond to changes in the environment, react to the unpredictability of business processes and act on opportunities when they arise [5].

Agent-Mediated Knowledge Management is a new research direction that aims at the cross-fertilization between the $\mathrm{KM}$ and the intelligent agent research fields [9]. Agentbased models for KM see agents as autonomous social entities (like employees in a company) that exhibit flexible, responsive and proactive behavior and the interactions among these entities give rise to complex dynamics. Current multi-agent models are not well suited for KM because they either take a centralistic approach to organizational design or have a purely emergent view on agent interactions. Collaboration support systems, as described above, require the integration of individual desires with organizational requirements. The multi-agent organizational model OperA presented in this paper incorporates formal organizational processes and goals and the different individual perspectives of the actors (people, groups and possibly systems) involved [4]. The purpose of this paper is to show the suitability of this model to describe and support knowledge sharing agreements.

\section{KNOWLEDGE SHARING}

One of the main aims of KM is to provide an environment for optimal sharing of knowledge between individuals and groups. Basically, knowledge sharing is done in two ways: by articulation and by socialization [7].

- Socialization is the sharing of tacit knowledge between people. In this way, knowledge moves from tacit to tacit. Knowledge does not become explicit and the organization as a whole cannot not easily use it.

- Articulation: An individual succeeds in formulating the fundaments of his/her own tacit knowledge in a way that can be stored or formalized. This process of making tacit knowledge explicit allows it to be shared within the organization.

Many current KM efforts focus on the articulation of knowledge, that is, in the conversion of tacit, personal knowledge into explicit, organizational knowledge. As anyone who has been involved in the development of expert systems and knowledge based systems (the traditional ways of formalizing knowledge) can tell, the cost of formalizing knowledge is very high and the resulting solution is not always very useful. The rate of usefulness (speed with which knowledge becomes obsolete or useless) and the probability of its reuse determine the benefit of formalizing a 'piece' of knowledge.

Socialization is often the preferred way of learning and sharing between people. The assumption that knowledge is inseparable from the communities that create it, use it, and transform it, motivates the use of Community of Practice
(CoP) concept in KM. A CoP is a group of people sharing a common area of expertise and/or who search for solutions to common problems [10]. A CoP is thus not necessarily an authorized or identified group. People in a community of practice can perform the same job, collaborate on a shared task or work together on a product. What holds them together is a common sense of purpose and a real need to know what each other knows. Most organizations will hold several communities of practice and most people belong to at least one of them [3].

\section{A KNOWLEDGE SHARING SCENARIO}

The Knowledge Center for Non-Life Insurance at a large insurance company in the Netherlands is responsible for the development and maintenance of the knowledge that will give business units across the organization a leading edge in the area. The center strives for efficient and goal directed sharing of information and knowledge. Members, which are insurance product developers and actuaries, are spread around the country. Their knowledge and expertise are greatly valuable and useful to each other. However, because people are not aware of each other's capabilities, often they will discuss their business problems with a direct colleague just because he/she happens to be conveniently close and not because he/she is the best person to consult with [1]. The objectives of the KennisNet project are to structure, initiate and organize the sharing of knowledge across the non-life development group and setting up a framework that assures the continuous availability of consistent and up-to-date knowledge.

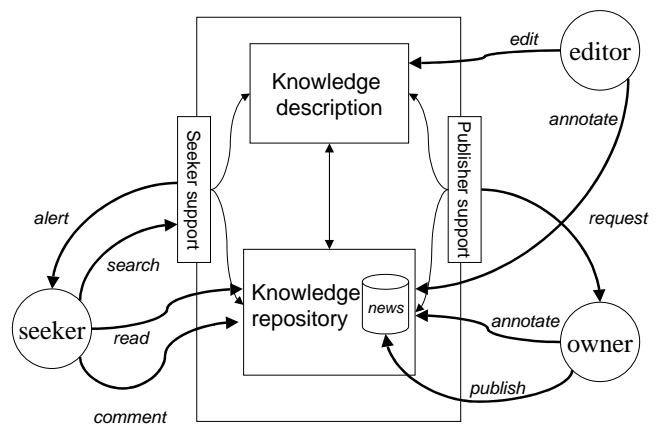

Figure 1; The architecture of KennisNet

The initial approach for the development of KennisNet incorporated direct contacts, organized as quarterly workshops with the participation of all members. In parallel to the workshops, a knowledge repository, depicted in Figure 1, was implemented to enable efficient distribution and maintenance of the group's knowledge. The repository was implemented using Lotus Notes.

After the knowledge repository was running for around one year, we conducted a user satisfaction survey, reported in [8]. Two main conclusions from this survey were that (1) the face-to-face structure was well appreciated and its value clear, but (2) the benefit and potential of the knowledge repository was not always clear to the users, and the repository was hardly used. 
The survey pointed out that the main reason for this lack of use is that users need a more personal means of interaction to make them comfortable exchanging knowledge. The survey also indicated that knowledge owners prefer to share their expertise within a controllable, trusted group under conditions negotiated for the specific situation and partners. The community of users supported by the KennisNet operates across business unit boundaries, independently of the holding organizational structure. Sharing knowledge therefore implies that the knowledge seeker and the knowledge owner must be able to find each other and agree on the terms of the exchange. The following requirements for an effective collaboration support system summarize the conclusions of the survey:

- Enable exchange within a controllable, trusted group under conditions negotiated by the partners for the specific situation

- Knowledge seekers and owners must be able to find each other and agree on the terms of the exchange.

In order to support the above collaboration requirements, the knowledge repository was extended with mechanisms for knowledge exchange and collaboration that keep ownership links between knowledge and people, for the support of negotiation and valuation of exchange conditions. Agents play here a useful role. As personal assistants, agents can ensure the preservation of individual needs and perspectives, and can be employed to monitor and assist knowledge exchange, for example by taking care that deadlines are kept, reports are effectively exchanged, and eventual changes are communicated. Furthermore, personal assistants can search the network for suitable partners, can publish results in the repository on behalf of their owners, and can monitor news and discussion groups.

These criteria motivated the choice for an agent-based implementation, using the OperA model [5]. The OperA approach consists of a 3-layered model that separates the concerns of the organization from those of the individual. The top layer, the Organizational Model (OM), describes the structure and objectives of a system as envisioned by the organization, in terms of roles and interactions. Individuals have however their own expectations and requirements that do not always fit organizational design. The middle layer, the Social Model (SM), describes the agent population in terms of the agreements made between individual agents, the personal assistants and the KennisNet agent society. Finally, the bottom layer, the Interaction Model (IM), describes specific agreements concerning interaction between agents.

\section{PERSONAL AGENTS FOR KNOWLEDGE SHARING}

In the following, we present the personalized knowledge sharing model for KennisNet, using the OperA framework.

\section{Organizational Model}

Besides the roles of seeker and owner that model the participants in KennisNet, the OM includes other roles that represent the organizational concerns. These roles ensure the realization of organizational requirements of sharing and distributing knowledge. Gatekeepers are responsible for accepting and introducing new agents to the society. Matchmakers keep track of agents, and of their needs and possibilities and mediate in the matching of demand and supply of knowledge. Notaries register and keep track of collaboration contracts between agents. Finally, monitoring agents are trusted third parties that keep track of the execution of collaboration contracts between agents.

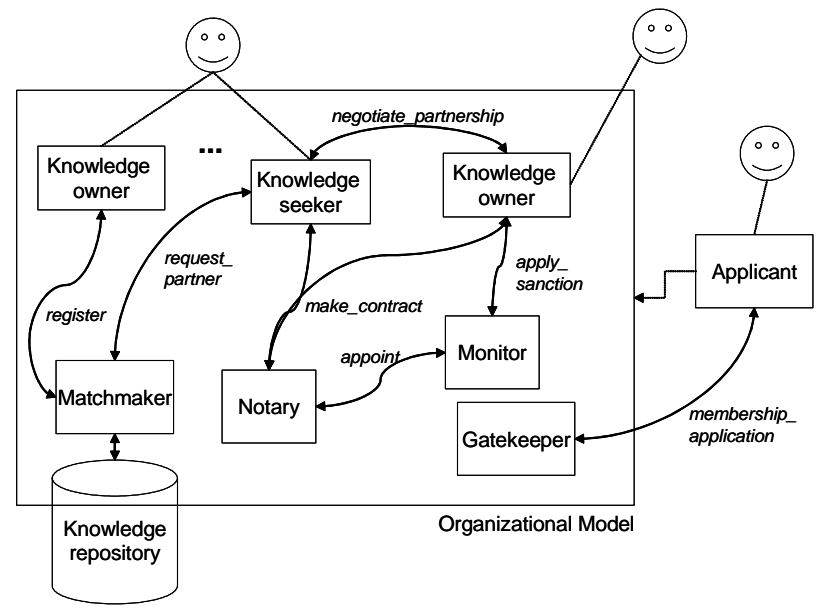

Figure 2: Agent-based architecture for KennisNet

Figure 2 depicts Organizational Model for KennisNet, including roles and interaction patterns.

\section{Social Model}

People seeking collaboration through the KennisNet initiate a personal agent that acts as their avatar in the system. This agent uses the preferences and conditions specified by the user to find appropriate partners and negotiate exchange terms. Depending on the specific task, the personal agent will take either the role of knowledge seeker or knowledge owner. Requirements concerning privacy, secrecy and competitiveness between brands and departments that influence the channels and possibilities of sharing are also described in the specification of the personal assistants. Typically in the KennisNet, members do not have restrictions concerning sharing of knowledge they bring in. However, especially when new products are concerned, it can happen that agents of members involved will require such knowledge to be shared only within a restricted group.

In the SM, social contracts describe the agreements between participating agents and the KennistNet. Negotiation of social contracts happens between the personal assistant and the Gatekeeper agent, who will watch over the interests of the society itself. For example, Anne is a member of the KennisNet group that is seeking knowledge on price policies from the competition. Anne will initiate an agent enacting the knowledge seeker role in the KennisNet. During the Start scene, the conditions for Anne's agent will be negotiated and fixed in a social contract. Such contract will, for instance, specify which parts of the repository Anne is allowed to access, which are the obligations of Anne concerning the publication of knowledge items received as result of an interaction, and 
whether Anne allows for items that she provides to be published or not.

\section{Interaction Model}

The IM describes agreements between agents by means of interaction contracts. In order to accommodate the autonomous behavior of agents, interaction contracts allow the specification of new, emergent, interaction paths, to the extent allowed by the organizational and social models.

The following example describes a contract between two members. In this example, which is fictive but typically possible in the domain of non-life insurance, Anne will provide Bob with a report about competition prices, on the condition that Bob will give her comments on the report (that she will have to present to her Unit directors) and eventually share with her his new pricing concept for car insurance. The notary agent registers the contract between Anne and Bob and assigns it a monitor agent that checks its fulfillment. Monitoring can be a very simple activity, where status is checked when a deadline is reached. However, we have chosen to use an agent as monitor because monitors can take a more active role, reminding parties of approaching deadlines or by suggesting possible actions when sanctions occur. In the case that one of the agents will not fulfill its commitments, sanctions will be applied. When sanctions are not explicitly specified in the contract, the norms of the society will be used. For instance, the Kennisnet follows the norm that agents that do not fulfill their commitments are given less priority in exchanges.

\section{CONCLUSIONS}

Collaboration management systems call for design approaches that are reactive and proactive in relation to the needs and expectations of its users. Agent concepts, which originated in artificial intelligence but which have further developed and evolved in many areas of computing, hold great promise for responding to the new realities of knowledge and collaboration management. In this paper, we have presented an agent-based model for organizations that fulfills the specification requirements of collaboration management systems. This model enables personalized support for knowledge sharing as it enables both knowledge seekers and owners to specify their own exchange conditions and functionalities.

Collaborative knowledge management environments are able to provide uniform access to a diversity of knowledge and information sources of different degree of formality and enable knowledge sharing in a personalized way. Personal assistant regulate the exchange activities between knowledge sources, including knowledge owners, and knowledge seekers.

Even though users are usually not concerned with the technology used to implement a system, the philosophy behind a technology determines for a great extent the way human-computer interaction occurs. In agent-oriented systems, interaction is intention or goal based instead of information or text-based. This drastically changes the way humans interact with computers. In the case of knowledge sharing, both in a repository as in a personal assistant support situation, the user will always provide its knowledge through the interface. However, what the user believes will happen with her input (that is, how it will be stored and with whom it will be shared) determine the degree of trust in the system. Initial results presented in this paper show that a more active participation of the user on the determination of exchange conditions influences positively her willingness to share. An important direction for HCI research is to investigate the influence of the intention of an interface on the actual interaction behavior

Finally, in the prototype implementation of KennisNet, the interface to a personal assistant was textual. Users could fill in a form indicating their knowledge needs or resources and their exchange requirements. Issues for future research include the study of the influence of human-agent interaction (e.g. textual interface vs. embodied agents) on trust and efficiency of exchanges between user and agent.

\section{REFERENCES}

1. Ahuja, M., Carley, K. 1998: Network Structure in Virtual Organizations, Journal of Computer Mediated Communication, 3(4), June.

2. Bonifacio, M., Bouquet, P., Traverso, P. 2002: Enabling Distributed Knowledge Management: Managerial and Technological Implications, Novatica and Informatik/Informatique, III (1).

3. Brown, J., Gray, E. 1995: The People are the Company. Fast Company, (1):78-82, Boston, MA. URL: http://www.fastcompany.com/online/01/people.html

4. Dignum, V. 2004: A Model for Organizational Interaction: Based on Agents, Founded in Logic. PhD thesis, Utrecht University.

5. Dignum, V., Dignum, F. 2003: The Knowledge Market: Agent Mediated Knowledge Sharing. Marik, V., Muller, J., Pechoucek, M. (Eds.): Proceedings of CEEMAS’03, Prague, June 16 - 18. LNAI, Springer-Verlag.

6. Eppler, M. 2002: Glossary definition: Knowledge management. NetAcademy: www.knowledgemedia.org.

7. Nonaka, I. 1991, “The Knowledge-Creating Company”, reprinted in 1998 in Harvard Business Review on Knowledge Management, Harvard Press.

8. Pumareja, D., Bondarouk, T., Sikkel, K. 2003: Supporting Knowledge Sharing Isn't Easy - Lessons Learnt from a Case Study. Information Resource Management Association International Conference (IRMA'03).

9. van Elst, L., Dignum, V., Abecker, A. 2003: Towards Agent-Mediated Knowledge Management. In: L. van Elst, V. Dignum, A. Abecker (Eds.): Agent-Mediated Knowledge Management: Selected Papers, LNAI 2926, Springer-Verlag,

10. Wenger, E., McDermott, R., Snyder, W. 2002: Cultivating Communities of Practice. Harvard Business School Press. 